\title{
A methodology for the design of robust rollover prevention controllers for automotive vehicles: Part 2-Active steering
}

\author{
Selim Solmaz*, Martin Corless and Robert Shorten
}

\begin{abstract}
In this paper we apply recent results from robust control to the problem of rollover prevention in automotive vehicles. Specifically, we exploit the results of Pancake, Corless and Brockman, which provide controllers to robustly guarantee that the peak magnitudes of the performance outputs of an uncertain system do not exceed certain values. We use the dynamic Load Transfer Ratio $L T R_{d}$ as a performance output for rollover prevention, and design active-steering based rollover controllers to keep the magnitude of this quantity below a certain level, while we use control input $u$ as an additional performance output to limit the maximum amount of control effort. We present numerical simulations to demonstrate the efficacy of our controllers.
\end{abstract}

\section{INTRODUCTION}

It is well known that vehicles with a high center of gravity such as vans, trucks, and the highly popular SUVs (Sport Utility Vehicles) are more prone to rollover accidents. According to the 2004 data [1], light trucks (pickups, vans, SUV's) were involved in nearly $70 \%$ of all the rollover accidents in the USA, with SUV's alone responsible for almost $35 \%$ of this total. The fact that the composition of the current automotive fleet in the U.S. consists of nearly $36 \%$ pickups, vans and SUV's [2], along with the recent increase in the popularity of SUV's worldwide, makes rollover an important safety problem.

There are two distinct types of vehicle rollover: tripped and untripped rollover. Tripped rollover is usually caused by impact of the vehicle with something else resulting in the rollover incident. Driver induced un-tripped rollover can occur during typical driving situations and poses a real threat for top-heavy vehicles. Examples are excessive speed during cornering, obstacle avoidance and severe lane change maneuvers, where rollover occurs as a direct result of the wheel forces induced during these maneuvers. It is however, possible to prevent such a rollover incident by monitoring the car dynamics and applying proper control effort ahead of time. Therefore there is a need to develop driver assistance technologies which would be transparent to the driver during normal driving conditions, but which act when needed to recover handling of the vehicle during extreme maneuvers [2].

We present in this paper a robust rollover prevention controller design methodology based on active steering. The proposed control design is an application of recent results on the design of control systems which guarantee that the peak value of the performance output of a plant does not exceed certain thresholds. [3]. The selected performance output for the rollover problem is the dynamic Load Transfer Ratio $L T R_{d}$. This measure of performance is related to tire lift-off and it can be considered as an early indicator of impending vehicle rollover. The aim of our control strategy is to limit the peak value of this performance output. The additional performance output on $u$ minimizes the maximum amount attenuation with the controller while achieving the objective performance on $L T R_{d}$. We

S. Solmaz (selim.solmaz@nuim.ie) and R. Shorten (robert.shorten@nuim.ie) are with the Hamilton Institute, National University of Ireland-Maynooth, Ireland. M. Corless (corless@purdue.edu) is with the School of Aeronautics \& Astronautics, Purdue University, West Lafayette, IN, USA.

*Corresponding author. Phone:+353 1 7086100, Fax: +353 17086269 indicate how our design can be extended to account for other sources of uncertainty such as unknown vehicle center of gravity, and tire stiffness parameters.

\section{RELATED WORK}

Rollover prevention is a topical area of research in the automotive industry (see, for example, http://www.safercar.gov/Rollover for a good introduction to the problem) and several studies have recently been published. Relevant publications include that of Palkovics et al. [4], where they proposed the ROP (Roll-Over Prevention) system for use in commercial trucks making use of the wheel slip difference on the two sides of the axles to estimate the tire lift-off prior to rollover. Wielenga [5] suggested the ARB (Anti Roll Braking) system utilizing braking of the individual front wheel outside the turn or the full front axle instead of the full braking action. The suggested control system is based on lateral acceleration thresholds and/or tire lift-off sensors in the form of simple contact switches. Chen et al. [6] suggested using an estimated TTR (Time To Rollover) metric as an early indicator for the rollover threat. When TTR is less than a certain preset threshold value for the particular vehicle under interest, they utilized differential breaking to prevent rollover. Ackermann et al. and Odenthal et al. [7], [8] proposed a robust active steering controller, as well as a combination of active steering and emergency braking controllers. They utilized a continuous-time active steering controller based on roll rate measurement. They also suggested the use of a static Load Transfer Ratio $\left(L T R_{S}\right)$ which is based on lateral acceleration measurement; this was utilized as a criterion to activate the emergency steering and braking controllers.

\section{Vehicle Modelling AND $L T R_{d}$}

We use a linearized vehicle model for control design. Specifically, we consider the well known single-track (bicycle) model with a roll degree of freedom. In this model the steering angle $\delta$, the roll angle $\phi$, and the vehicle sideslip angle $\beta$ are all assumed to be small. We further assume that all the vehicle mass is sprung, which implies insignificant wheel and suspension weights. The lateral forces on the front and rear tires, denoted by $S_{v}$ and $S_{h}$, respectively, are represented as linear functions of the tire slip angles $\alpha_{v}$ and $\alpha_{h}$, that is, $S_{v}=C_{v} \alpha_{v}$ and $S_{h}=C_{h} \alpha_{h}$, where $C_{v}$ and $C_{h}$ are the front and rear tire stiffness parameters respectively. In order to simplify the model description, we further define the following auxiliary variables

$$
\begin{aligned}
& \sigma \triangleq C_{v}+C_{h}, \\
& \rho \triangleq C_{h} l_{h}-C_{v} l_{v}, \\
& \kappa \triangleq C_{v} l_{v}^{2}+C_{h} l_{h}^{2},
\end{aligned}
$$

where $l_{v}$ and $l_{h}$ are defined in Figure 1. For simplicity, it is assumed that, relative to the ground, the sprung mass rolls about a horizontal roll axis which is along the centerline of the body and at ground level. Using the parallel axis theorem of mechanics, $J_{x_{e q}}$, 

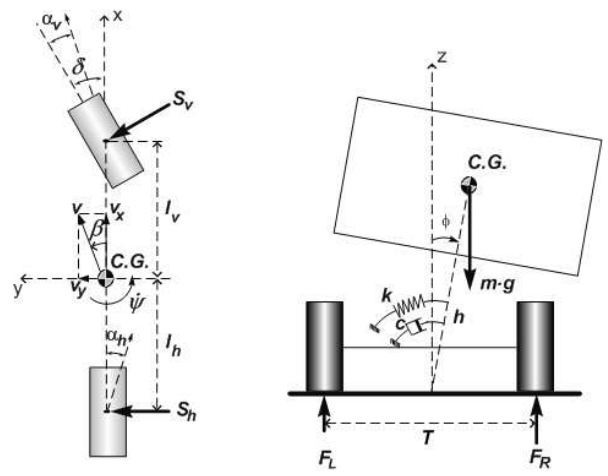

Fig. 1. Linear bicycle model with roll degree of freedom.

TABLE I

MODEL PARAMETERS AND THEIR DEFINITIONS

\begin{tabular}{cll}
\hline Parameter & Description & Unit \\
\hline$m$ & vehicle mass & {$[\mathrm{kg}]$} \\
$v$ & vehicle speed & {$[\mathrm{m} / \mathrm{s}]$} \\
$\delta$ & steering angle & {$[\mathrm{rad}]$} \\
$J_{x x}$ & roll moment of inertia at CG & {$\left[\mathrm{kg} \cdot \mathrm{m}^{2}\right]$} \\
$J_{z z}$ & yaw moment of inertia at CG & {$\left[\mathrm{kg} \cdot \mathrm{m}^{2}\right]$} \\
$l_{v}$ & longitudinal CG position w.r.t. front axle & {$[\mathrm{m}]$} \\
$l_{h}$ & longitudinal CG position w.r.t. rear axle & {$[\mathrm{m}]$} \\
$T$ & vehicle track width & {$[\mathrm{m}]$} \\
$h$ & distance of CG from roll axis & {$[\mathrm{m}]$} \\
$c$ & suspension damping coefficient & {$[\mathrm{N} \cdot \mathrm{m} \cdot \mathrm{s} / \mathrm{rad}]$} \\
$k$ & suspension spring stiffness & {$[\mathrm{N} \cdot \mathrm{m} / \mathrm{rad}]$} \\
$C_{v}$ & linear tire stiffness for front tire & {$[\mathrm{N} / \mathrm{rad}]$} \\
$C_{h}$ & linear tire stiffness for rear tire & {$[\mathrm{N} / \mathrm{rad}]$} \\
\hline
\end{tabular}

the moment of inertia of the vehicle about the assumed roll axis, is given by

$$
J_{x_{e q}}=J_{x x}+m h^{2}
$$

where $h$ is the distance between the center of gravity (CG) and the assumed roll axis and $J_{x x}$ is the moment of inertia of the vehicle about the roll axis through the CG. We introduce the state vector $\xi=\left[\begin{array}{llll}v_{y} & \dot{\psi} & \dot{\phi} & \phi\end{array}\right]^{T}$, where descriptions are as follows:

$v_{y}$ : lateral velocity of the $\mathrm{CG}$

$\psi$ : yaw rate of the unsprung mass

$\dot{\phi}$ : roll rate of the sprung mass

$\phi$ : roll angle of the sprung mass

The equations of motion corresponding to this model are as follows:

$$
\dot{\xi}=A \xi+B \delta
$$

where

$$
\begin{aligned}
& A=\left[\begin{array}{cccc}
-\frac{\sigma J_{x e q}}{m v J_{x x}} & \frac{\rho J_{x e q}}{m v J_{x x}}-v & -\frac{h c}{J_{x x}} & \frac{h(m g h-k)}{J_{x x}} \\
\frac{\rho}{J_{z z} v} & -\frac{\kappa}{J_{z z} v} & 0 & 0 \\
-\frac{h \sigma}{J_{x x} v} & \frac{h \rho}{v J_{x x}} & -\frac{c}{J_{x x}} & \frac{m g h-k}{J_{x x}} \\
0 & 0 & 1 & 0
\end{array}\right], \\
& B=\left[\begin{array}{llll}
\frac{C_{v} J_{x e q}}{m J_{x x}} & \frac{C_{v} l_{v}}{J_{z z}} & \frac{h C_{v}}{J_{x x}} & 0
\end{array}\right]^{T} .
\end{aligned}
$$

Further definitions of the parameters appearing in (4) and (5) are given in Table I. Also see [9] for a detailed description and derivation of this vehicle model.

\section{A. The Load Transfer Ratio, $L T R_{d}$}

Traditionally, as discussed in the related work section, some estimate of the vehicle load transfer ratio (LTR) has been used as a basis for the design of rollover prevention systems. The quantity LTR [8], [10] can be simply defined as the load (i.e., vertical force) difference between the left and right wheels of the vehicle, normalized by the total load (i.e., the weight of the car). In other words

$$
L T R=\frac{\text { Load on Right Tires-Load on Left Tires }}{\text { Total Weight }} .
$$

It is apparent that $L T R$ varies within $[-1,1]$, and for a perfectly symmetric car that is driving straight, it is 0 . The extrema are reached in the case of a wheel lift-off of one side of the vehicle, in which case $L T R$ becomes 1 or -1 depending on the side that lifts off. If roll dynamics are ignored, it is easily shown [8] that the corresponding static LTR (which we denote by $L T R_{S}$ ) is approximated by

$$
L T R_{s} \triangleq \frac{2 a_{y}}{g} \frac{h}{T}
$$

where $a_{y}$ is the lateral acceleration of the CG.

Note that rollover estimation based upon (7) is not sufficient to detect the transient phase of rollover (due to the fact that it is derived ignoring roll dynamics). Consequently, we obtain an expression for LTR which does not ignore roll dynamics. We denote this by $L T R_{d}$. In order to derive $L T R_{d}$ we write a torque balance equation. Recall that we assumed the unsprung mass to be insignificant and that the main body of the vehicle rolls about an axis along the centerline of the body at the ground level. We can write a torque balance for the unsprung mass about the assumed roll axis in terms of the suspension torques and the vertical wheel forces as follows:

$$
-F_{R} \frac{T}{2}+F_{L} \frac{T}{2}-k \phi-c \dot{\phi}=0 .
$$

Now substituting the definition of $L T R$ from (6) and rearranging yields the following expression for $L T R_{d}$ :

$$
L T R_{d}=-\frac{2}{m g T}(c \dot{\phi}+k \phi) .
$$

In terms of the state vector, $L T R_{d}$ can be represented by the following linear matrix equation

$$
\begin{aligned}
L T R_{d} & =C \xi, \\
C & =\left[\begin{array}{llll}
0 & 0 & -\frac{2 c}{m g T} & -\frac{2 k}{m g T}
\end{array}\right] .
\end{aligned}
$$

\section{B. Actuators, Sensors and Parameters}

We are interested in robust control design based on active steering actuators. There are two types of active steering methods: full steerby-wire and mechatronic-angle-superposition types. Steer-by-wire actuators do not contain a physical steering column between the steering wheel and the tires, which enable them to be flexible and suitable for various vehicle dynamics control applications. However, stringent safety requirements on such systems prevent them from entering today's series-production vehicles. Mechatronicangle-superposition type active steering actuators however have been recently introduced to the market. They contain a physical steering column and act cooperatively with the driver, while they permit various functions such as speed dependent steering ratio modification, and active response to mild environmental disturbances. It is plausible that active steering actuators will become an industry standard in the near future, due to their capability of directly and most efficiently affecting the lateral dynamics of the 
car. Active steering based lateral control methods can be perfectly transparent to the driver and they are likely to cause the least interference with the vehicle response and the driver intent, unlike the control approaches based on differential braking and active suspension that can abruptly affect the vehicle response during dangerous maneuvers. The biggest factor in this is the fact that use of active steering actuators do not result in a significant velocity loss, and for this reason they are likely to enter the market initially for the high performance vehicle segment. Therefore, in this paper we assume mechatronic-angle-superposition type steering actuators; however results can easily be extended to the use of steer-by-wire actuators.

We also assume full state feedback information for the design of the reference robust controllers and that all the model parameters $m, J_{x x}, J_{z z}, l_{v}, l_{h}, C_{v}, C_{h}, k, h, c$ are known. This is an unrealistic assumption: yet our control design is easily extended to account for uncertainty in these parameters. As a side note, although we assumed all the vehicle model parameters to be known, it is possible to estimate some of these that are fixed (but unknown) using the sensor information available for the control design suggested here; this however is outside the scope of this work [11].

\section{State Feedback Controllers for Robust Disturbance ATtENUATION}

We are interested in designing a controller to prevent rollover that is robust with respect to parameter uncertainty. Our starting point is in results obtained by Pancake, Corless and Brockman [3], [12] for uncertain systems of the form

$$
\begin{aligned}
\dot{x} & =A(\theta) x+B(\theta) \omega+B_{u}(\theta) u \\
z_{j} & =C_{j}(\theta) x+D_{j}(\theta) \omega+D_{j u}(\theta) u,
\end{aligned}
$$

where $\theta$ is some parameter that captures the plant nonlinearity/uncertainty, $x \in \mathbb{R}^{n}$ is the state at time $t \in \mathbb{R}$ and $\omega \in \mathbb{R}$ is a bounded disturbance input while $z_{j} \in \mathbb{R}$ are the performance outputs for $j=1, \ldots, r$. We wish to synthesize a stabilizing controller which prevents the peak value of the performance outputs exceeding a certain value. In other words, we want to design a feedback controller, which guarantees bounded performance outputs given a bounded uncertain disturbance, that is, $\|\omega\| \leq \omega_{\max }$. In order to keep the problem simple, we consider linear state feedback controllers of the form

$$
u=K x,
$$

where $K$ is a constant matrix. We can now define closed loop system matrices $A_{c l}$ and $C_{c l_{j}}$ as follows

$$
A_{c l}(\theta)=A(\theta)+B_{u}(\theta) K, \quad C_{c l_{j}}(\theta)=C_{j}(\theta)+D_{j u}(\theta) K,
$$

for all $j=1, \ldots, r$. Applying (13) to system (11)-(12) and using the closed loop matrix definitions (14) we obtain the following closed loop system:

$$
\begin{aligned}
\dot{x} & =A_{c l}(\theta) x+B(\theta) \omega \\
z_{j} & =C_{c l_{j}}(\theta) x+D_{j}(\theta) \omega, \quad j=1, \ldots, r .
\end{aligned}
$$

Assumption 1: For each $j=1, \ldots, r$, and $\theta$, the matrix

$$
\left[\begin{array}{llllll}
A(\theta) & B(\theta) & B_{u}(\theta) & C_{j}(\theta) & D_{j}(\theta) & D_{j u}(\theta)
\end{array}\right]
$$

can be written as a convex combination of a finite number of matrices

$$
\left[A_{1} B_{1} B_{u 1} C_{j 1} D_{j 1} D_{j u 1}\right], \ldots,\left[A_{N} B_{N} B_{u N} C_{j N} D_{j N} D_{j u N}\right],
$$

that is, for each $\theta$ there exists non-negative scalars $\xi_{1}, \ldots, \xi_{N}$ such that $\sum_{i=1}^{N} \xi_{i}=1$, and

$$
\begin{aligned}
A(\theta) & =\sum_{i=1}^{N} \xi_{i} A_{i}, \quad C_{j}(\theta)=\sum_{i=1}^{N} \xi_{i} C_{j i}, \\
B(\theta) & =\sum_{i=1}^{N} \xi_{i} B_{i}, \quad B_{u}(\theta)=\sum_{i=1}^{N} \xi_{i} B_{u i}, \\
D_{j}(\theta) & =\sum_{i=1}^{N} \xi_{i} D_{j i}, \quad D_{j u}(\theta)=\sum_{i=1}^{N} \xi_{i} D_{j u i} .
\end{aligned}
$$

We have now the following result which is useful for control design.

Theorem 1: Consider a nonlinear/uncertain system described by (11)-(12) and satisfying Assumption 1. Suppose that there exists a matrix $S=S^{T}>0$, a matrix $L$ and positive scalars $\beta_{1}, \ldots \beta_{N}$ and $\mu_{j, 0}, \mu_{j, 1}, \mu_{j, 2}$ such that for each $j=1, \ldots, r$ the following matrix inequalities hold

$$
\begin{array}{r}
{\left[\begin{array}{cc}
\beta_{i}\left(S A_{i}^{T}+A_{i} S+L^{T} B_{u i}^{T}+B_{u i} L\right)+S & \beta_{i} B_{i} \\
\beta_{i} B_{i}^{T} & -\mu_{j 0} I
\end{array}\right] \leq 0,} \\
{\left[\begin{array}{ccr}
-\mu_{j 1} S & 0 & S C_{j i}^{T}+L^{T} D_{j u i}^{T} \\
0 & -\mu_{j 2} I & D_{j i}^{T} \\
C_{j i} S+D_{j u i} L & D_{j i} & -I
\end{array}\right] \leq 0,}
\end{array}
$$

for all $i=1, \ldots, N$. Then the controller

$$
u=K x, \quad \text { where } K=L S^{-1}
$$

results in a closed loop nonlinear/uncertain system (15)-(16) which is $L_{\infty}$ stable with $L_{\infty}$ gains less than or equal to

$$
\gamma_{j}=\sqrt{\mu_{j 0} \mu_{j 1}+\mu_{j 2}} .
$$

The above means that for a bounded disturbance input, that is, $\|\omega(t)\| \leq \omega_{\max }$ for all $t$, and zero initial state, the performance outputs $z_{1}, \ldots, z_{r}$ of the closed loop system are bounded and satisfy $\left\|z_{j}(t)\right\| \leq \gamma_{j} \omega_{\max }$ for all $t$. The scalars $\gamma_{1}, \ldots \gamma_{r}$ are called levels of performance and can be regarded as measures of the ability of the closed loop system to attenuate the effect of the disturbance input on the performance outputs; a smaller $\gamma_{j}$ means better performance in the sense of increased attenuation. For a proof of the theorem, see [12].

\section{Rollover Prevention CONTROLlers}

Here we use the results of the previous section to obtain robust rollover prevention controllers using active steering as the sole control input.

For the implementation of an active steering state feedback controller, we used the reference model (3) along with an additional control input term that is superimposed on the driver steering input (i.e., disturbance input); this is described by

$$
\dot{\xi}=A \xi+B \omega+B u,
$$

where $\xi(t) \in \mathbb{R}^{4}$ is the state at time $t \in \mathbb{R}$, and fixed matrices $A$ and $B$ are described as in (4) and (5). Here $u(t) \in \mathbb{R}$ is the control input and $\omega(t) \in \mathbb{R}$ denotes the disturbance input. In this paper we designate the driver commanded input $\delta_{d}$ to be a disturbance input and active steering input $\delta_{c}$ as the control input. i.e.,

$$
\begin{aligned}
\omega & =\delta_{d} \\
u & =\delta_{c},
\end{aligned}
$$

where the total steering angle is the sum of these two inputs such that $\delta=\delta_{c}+\delta_{d}$. Note that this is where we make use of the mechatronic-angle-superposition type steering actuators. For 
this problem we a considered proportional-integral (PI) type state feedback controller of the form

$$
u=K_{P} \xi+K_{I} \xi_{I}
$$

where the integrator state $\xi_{I}$ is the integral of the yaw rate tracking error with a zero initial condition, that is,

$$
\dot{\xi}_{I}=\dot{\psi}-\dot{\psi}_{\text {ref }}, \quad \xi_{I}(0)=0 .
$$

The reference yaw rate $\dot{\psi}_{\text {ref }}$ is the steady yaw rate which results from a constant driver input $\delta_{d}$ and zero control input; thus

$$
\dot{\psi}_{\text {ref }}=\alpha \delta_{d},
$$

for a constant gain $\alpha$. The above control structure is schematically depicted on Figure 2 below.

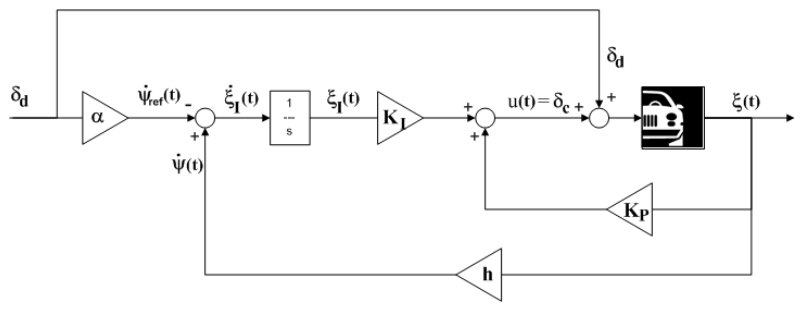

Fig. 2. Flow diagram of the PI active steering controller.

We can describe the system resulting from (22), (26) and (27) by

$$
\begin{aligned}
\dot{\xi} & =A \xi+B \delta_{d}+B u \\
\dot{\xi}_{I} & =\dot{\psi}-\alpha \delta_{d} .
\end{aligned}
$$

We introduce the performance outputs $z_{1}, z_{2}$ which are the $L T R_{d}$ given by (9) that helps in detecting the rollover likelihood, and the control effort $u$ that enables us to bound the maximum control effort. We are interested in synthesizing a $L_{\infty}$ stabilizing controller with closed loop performance measures $\gamma_{1}$ and $\gamma_{2}$ for $z_{1}$ and $z_{2}$, respectively. These performance outputs can be expressed as follows:

$$
\begin{aligned}
& z_{1}=C \xi \\
& z_{2}=u
\end{aligned}
$$

where $C$ is given as in (9). We can now define a new augmented state $x=\left[\begin{array}{ll}\xi^{T} & \xi_{I}\end{array}\right]^{T}$ and express (28)-(31) as

$$
\begin{aligned}
\dot{x} & =\tilde{A} x+\tilde{B} \delta_{d}+\tilde{B_{u}} u \\
z_{1} & =\tilde{C_{1}} x \\
z_{2} & =u
\end{aligned}
$$

with

$$
\begin{aligned}
\tilde{A} & =\left[\begin{array}{ll}
A & 0 \\
h & 0
\end{array}\right], \tilde{B}=\left[\begin{array}{c}
B \\
-\alpha
\end{array}\right], \tilde{B_{u}}=\left[\begin{array}{l}
B \\
0
\end{array}\right] \\
\tilde{C}_{1} & =\left[\begin{array}{ll}
C & 0
\end{array}\right],
\end{aligned}
$$

where $h=\left[\begin{array}{llll}0 & 1 & 0 & 0\end{array}\right]$. Also, the proposed controller structure (25) can be described by $u=K x$ where

$$
K=\left[\begin{array}{ll}
K_{P} & K_{I}
\end{array}\right] .
$$

We used Theorem 1 to design an $L_{\infty}$ controller with performance levels $\gamma_{j}$ where $j=1,2$. In our simulations the model parameters for (22) were chosen to approximate the behavior of a compact class vehicle. The choice of the compact class vehicle was totally
TABLE II

MODEL PARAMETERS

\begin{tabular}{cll}
\hline parameter & value & unit \\
\hline$m$ & 1224 & {$[\mathrm{~kg}]$} \\
$J_{x x}$ & 362.6 & {$\left[\mathrm{~kg} \cdot \mathrm{m}^{2}\right]$} \\
$J_{z z}$ & 1280 & {$\left[\mathrm{~kg} \cdot \mathrm{m}^{2}\right]$} \\
$l_{v}$ & 1.102 & {$[\mathrm{~m}]$} \\
$l_{h}$ & 1.25 & {$[\mathrm{~m}]$} \\
$T$ & 1.51 & {$[\mathrm{~m}]$} \\
$h$ & 0.375 & {$[\mathrm{~m}]$} \\
$c$ & 4000 & {$[\mathrm{~N} \cdot \mathrm{m} \cdot \mathrm{s} / \mathrm{rad}]$} \\
$k$ & 36075 & {$[\mathrm{~N} \cdot \mathrm{m} / \mathrm{rad}]$} \\
$C_{v}$ & 90240 & {$[\mathrm{~N} / \mathrm{rad}]$} \\
$C_{h}$ & 180000 & {$[\mathrm{~N} / \mathrm{rad}]$} \\
\hline
\end{tabular}

arbitrary and the results can easily extended to other class of vehicles with higher CG positions. The parameters used for the simulation are given in Table II and state responses to a step steering input and zero control input are shown in Figure 3. These state responses correspond to $\delta_{d}=30^{\circ}$ driver step steering input (where the steering ratio was assumed to be 1:17.5) and vehicle speed was chosen as $v=40 \mathrm{~m} / \mathrm{s}$.

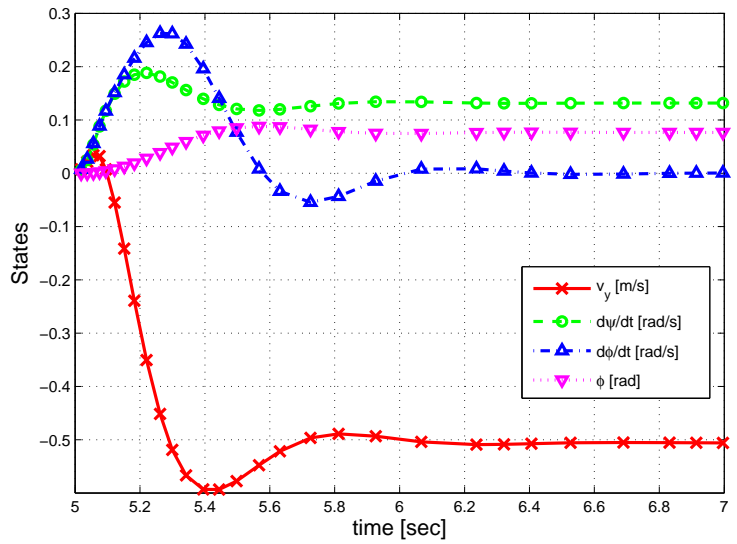

Fig. 3. State responses to a step steering input.

In order to find controller gain matrices $K_{P}$ and $K_{I}$ so that the resulting closed loop system has desirable performance, we used an iterative solution algorithm based on the one described in [3], [12] to obtain solutions to the matrix inequalities of Theorem 1. We attempted to minimize the level of performance $\gamma_{1}$ for a specified level of performance $\gamma_{2}$. In the numerical simulations we simulated an obstacle avoidance maneuver that is known as the elk-test, which takes place at a speed of $v=40 \mathrm{~m} / \mathrm{s}$ and a peak driver steering magnitude of $100^{\circ}$. The results are presented in Figures 4-9, which demonstrates the effectiveness of the controller. Specifically, in Figure 4 we compare the $L T R_{d}$ for the vehicles with and without rollover prevention control and observe that the vehicle with feedback achives the design objective of keeping $L T R_{d}$ value within the permissible bounds and prevents rollover. In Figure 6 we compare the driver steering input, controller steering input and the resultant steering input, which is the superposition of last two. We observe in this plot how the control actuator reacts suddenly at the start of the manuever and then settles down as the rollover threat is reduced. In Figures 5 and 7 we compare the corresponding roll angle and yaw rate variations during this manuever. 


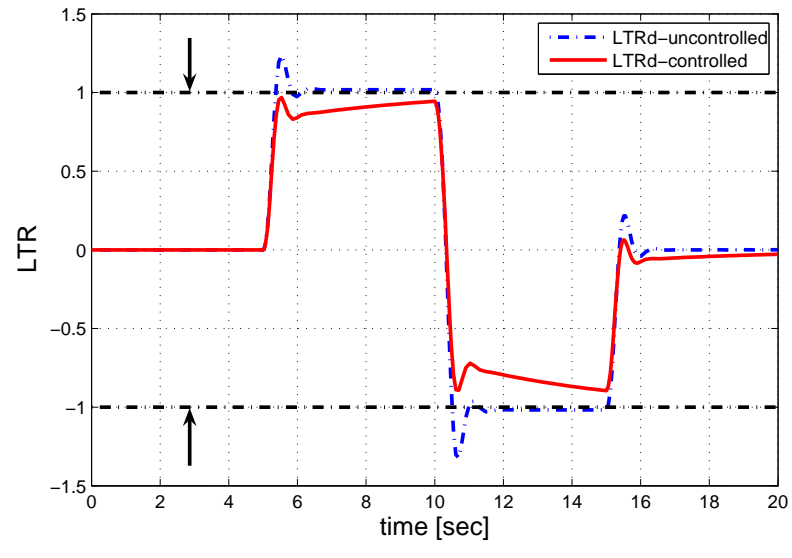

Fig. 4. Comparison of $L T R_{d}$ for the controlled and uncontrolled vehicles.

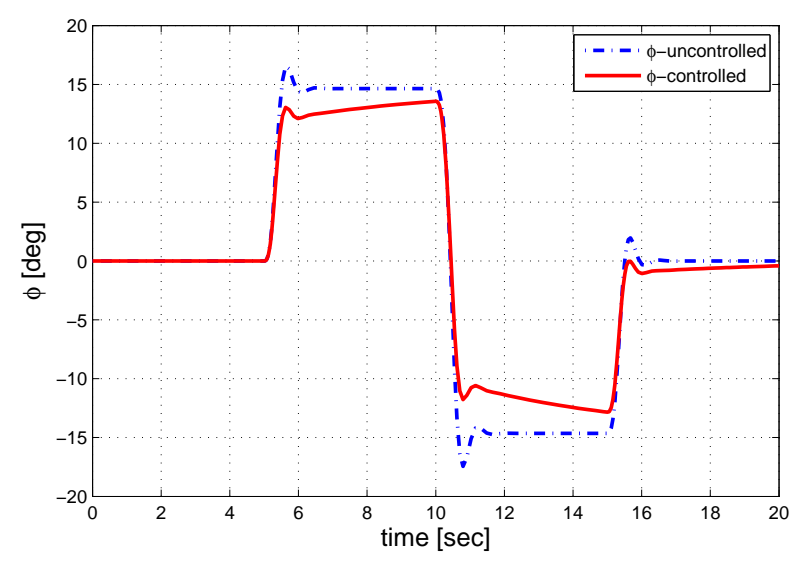

Fig. 5. Comparison of $\phi$ for the controlled and uncontrolled vehicles.

In figure 8 we compare the lateral velocities for the controlled and uncontrolled vehicles and observe that the controlled vehicle has a significant drop in the peak magnitude of lateral speed. Also note that sideslip variations can be obtained easily by normalizing the lateral velocities by longitudinal speed, which is assumed to be constant for this simulation. Finally in Figure 9 we compare the inertial trajectories corresponding to vehicles with and without the rollover prevention controller, and both with zero initial position.

Comment-1: In the presented control design we assume no parameter uncertainties.

Comment-2: Our design is easily extended to incorporate compensation for parameter uncertainties such as the unknown vehicle parameters, velocity variations, unknown mass and center of gravity height as presented in recent publications [13], [14].

Comment-3: A basic problem with the controller design method introduced here is that the controller is always active. That is, it will always attempt to limit the LTR, even in non-critical situations, thus potentially interfering with, and annoying the vehicle driver. It therefore makes sense to activate the controller in situations only when the potential for rollover is significant. In [14] such a switching criteria for activating the controller based on Lyapunov theory is given, which works in conjunction with the design method introduced in this paper.

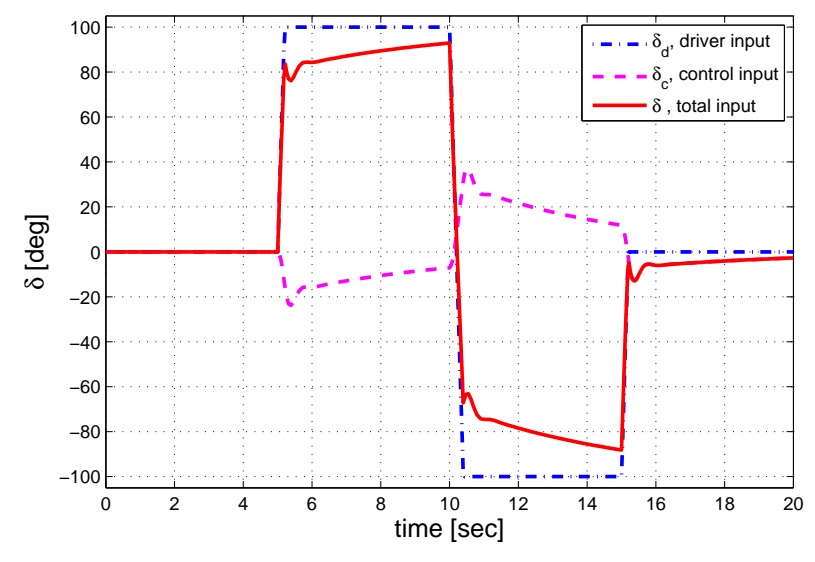

Fig. 6. Comparison of the steering commands and resulting steering angle.

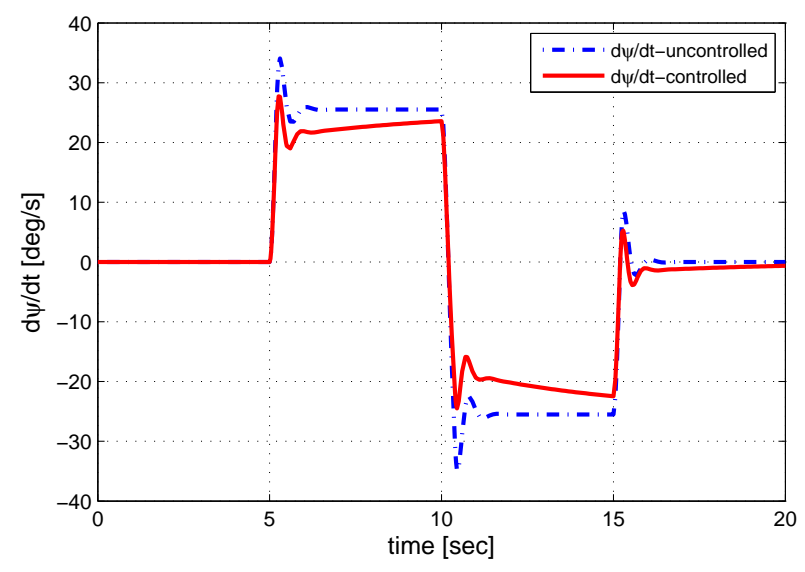

Fig. 7. Comparison of yaw rate for the controlled and uncontrolled vehicles.

\section{CONCLUSiOnS}

We have presented a methodology for the design of vehicle rollover prevention systems using differential braking. By introducing the load transfer ratio $L T R_{d}$, we obtain a system performance output whose value provides an accurate measure for determining the onset of rollover. Our rollover prevention system is based upon recent results from Pancake, Corless and Brockman, which provide controllers to robustly guarantee that the peak value of the performance outputs of an uncertain system do not exceed a certain value. Simulation results are presented to illustrate the benefits of the proposed approach. Future work will proceed in several directions. We shall extend the methodology to include differential braking, active suspension and combinations thereof to refine our rollover prevention strategy. We shall also examine the efficacy of our controllers in the presence of conditions which can result in a tripped rollover. A second strand of work will investigate refinement of the synthesis procedure. In particular, we shall investigate whether feasibility conditions can be developed to determine the existence of control gains to achieve certain pre-specified performance parameters $\gamma_{j}$. We will also look at introducing tire nonlinearities into the models for more realistic vehicle behavior. Finally, we hope to implement and evaluate our control system in real production vehicles in collaboration with our 


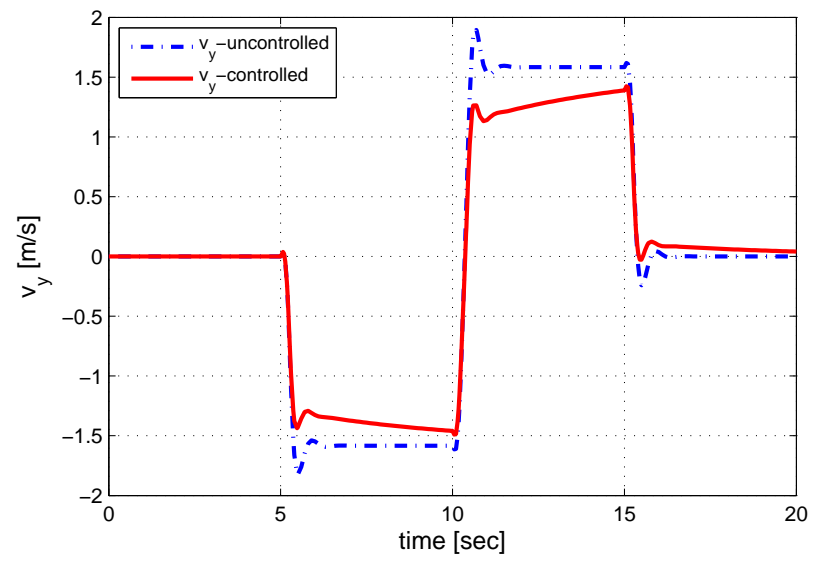

Fig. 8. Comparison of lateral velocity for the controlled and uncontrolled vehicles.

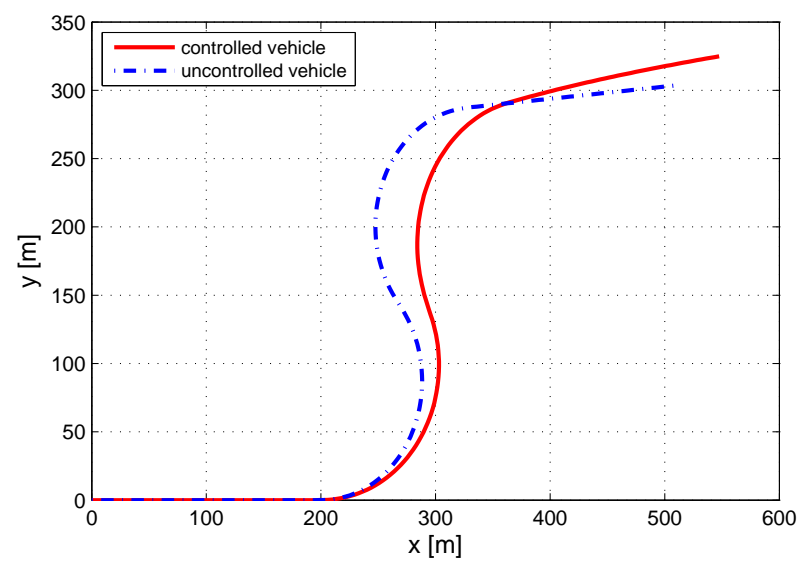

Fig. 9. Comparison of inertial trajectories for the controlled and uncontrolled vehicles starting from origin.

industrial partners.

\section{ACKNOWLEDGEMENTS}

This work was partially supported by Science Foundation Ireland Grant 04/IN3/I478.

\section{REFERENCES}

[1] National Highway Trafic Safety Administration (NHTSA), Trafic Safety Facts 2004: A Compilation of Motor Vehicle Crash Data from the Fatality Analysis Reporting System and the General Estimates System,Technical Report, 2006.

[2] Carlson C.R. and Gerdes J.C., "Optimal Rollover Prevention with Steer by Wire and Differential Braking",Proceedings of ASME International Mechanical Engineering Congress and Exposition, IMECE'03, Washington, D.C.,November 16-21,2003.

[3] Pancake T., Corless M., and Brockman M., "Analysis and Control of Polytopic Uncertain/Nonlinear Systems in the Presence of Bounded Disturbance Inputs", Proceedings of the American Control Conference, Chicago, IL, June 2000.

[4] Palkovics L., Semsey À. and Gerum E., "Roll-Over Prevention System for Commercial Vehicles-Additional Sensorless Function of the Electronic Brake System”, Vehicle System Dynamics, 1999, Vol.4, pp.285-297.

[5] Wielenga T.J., "A Method for Reducing On-Road Rollovers: Anti -Rollover Braking", SAE Paper No. 1999-01-0123, 1999.
[6] Chen B. and Peng H.,"Differential-Breaking-Based Rollover Prevention for Sport Utility Vehicles with Human-in-the-loop Evaluations",Vehicle System Dynamics,36(4-5):359-389,2001.

[7] Ackermann J. and Odenthal D.,"Robust steering control for active rollover avoidance of vehicles with elevated center of gravity",Proceedings of International Conference on Advances in Vehicle Control and Safety,Amiens, France, July 1998.

[8] Odenthal D., Bünte T. and Ackermann J.,"Nonlinear steering and breaking control for vehicle rollover avoidance",Proceedings of European Control Conference, Karlsruhe, Germany, 1999.

[9] Kiencke U. and Nielsen L., Automotive Control Systems for Engine, Driveline and Vehicle, Springer-Verlag \& SAE Int., Berlin, 2000.

[10] Kamnik R., Böttiger F., Hunt K., "Roll Dynamics and Lateral Load Transfer Estimation in Articulated HeavyFreight Vehicles: A Simulation Study", Proceedings of the Institution of Mechnaical Engineers, Part D, 2003.

[11] Akar M., Solmaz S. and Shorten R., "Method for Determining the Center of Gravity for an Automotive Vehicle", 2006, Irish Patent.

[12] Pancake T., Corless M., and Brockman M., "Analysis and Control of a Class of Uncertain/Nonlinear Systems in the Presence of Bounded Disturbance Inputs", In Preperation.

[13] Solmaz S., Corless M., and Shorten R., "A methodology for the design of robust rollover prevention controllers for automotive vehicles: Part 1-Differential Braking", Proceedings of the Conference on Decision and Control, San Diego, 2006.

[14] Solmaz S., Corless M., and Shorten R., "A Methodology for the Design of Robust Rollover Prevention Controllers for Automotive Vehicles with Active Steering", International Journal of Control, Special Issue on Automotive Systems and Control, to appear, 2007. 